\title{
Higgs production in heavy-quark annihilation through next-to-next-to-leading order QCD
}

\author{
Robert V. Harlander ${ }^{\mathrm{a}}$ \\ Institute for Theoretical Particle Physics and Cosmology, RWTH Aachen University, Aachen 52056, Germany
}

Received: 16 December 2015 / Accepted: 12 April 2016 / Published online: 2 May 2016

(C) The Author(s) 2016. This article is published with open access at Springerlink.com

\begin{abstract}
The total inclusive cross section for charged and neutral Higgs production in heavy-quark annihilation is presented through NNLO QCD. It is shown that, aside from an overall factor, the partonic cross section is independent of the initial-state quark flavors, and that any interference terms involving two different Yukawa couplings vanish. A simple criterion for defining the central renormalization and factorization scale is proposed. Its application to the $b \bar{b} \phi$ process yields results which are compatible with the values usually adopted for this process. Remarkably, we find little variation in these values for the other initial-state quark flavors. Finally, we disentangle the impact of the different parton luminosities from genuine hard NNLO effects and find that, for the central scales, a naive rescaling by the parton luminosities approximates the full result remarkably well.
\end{abstract}

\section{Introduction}

Models with an extended Higgs sector typically predict a spectrum of Higgs bosons with very diverse properties (see, e.g., [1]). This means that the relative importance of individual processes for the total production cross section can be very different compared to the Standard Model (SM) Higgs boson $H$, where the main contribution to the total cross section at the Large Hadron Collider (LHC) is given by gluon fusion, $g g \rightarrow H$ (see, e.g., [2-4]). In particular, quark-associated production can be much more important than for SM Higgs production. For example, in supersymmetric theories, it can naturally happen that at least one of the neutral Higgs bosons $\phi$ would be predominantly produced at the LHC in association with bottom quarks, $p p \rightarrow$ $\phi b \bar{b}$. Also an enhanced coupling to charm quarks can occur in many beyond-the-SM (BSM) scenarios, leading to nonnegligible contributions of associated Higgs-charm production [5]. Similarly, the cross section for charged Higgs bosons

\footnotetext{
a e-mail: robert.harlander@cern.ch
}

$\phi^{ \pm}$may receive contributions from associated $c \bar{s} \phi^{-} / \bar{c} s \phi^{+}$or $c \bar{b} \phi^{-} / \bar{c} b \phi^{+}$production, and we could even imagine flavorviolating contributions of the form $(b \bar{s}+\bar{b} s) \phi$ to neutral Higgs production [6].

The proper theoretical description of associated $b \bar{b} \phi$ production has a long and still ongoing history. The main argument has been centered around the question whether the so-called 4- or 5-flavor scheme (referred to as 4FS or 5FS in the following) is more appropriate to obtain the best approximation of the total inclusive cross section. In the 4FS, bottom-quark parton densities are neglected, so that the dominant leadingorder (LO) partonic process for $b \bar{b} \phi$ production is $g g \rightarrow b \bar{b} \phi$ (the cross section for $q \bar{q} \rightarrow b \bar{b} \phi$ is about a factor of ten smaller at the LHC). Integration over the final-state bottomquark momenta leads to logarithms of the form $\ln \left(m_{b} / m_{\phi}\right)$ in the total inclusive Higgs production cross section, where $m_{b}$ and $m_{\phi}$ is the bottom-quark and the Higgs-boson mass, respectively. The 5FS resums these terms to all orders in the strong coupling $\alpha_{s}$ by introducing bottom-quark parton densities, and describing the LO partonic cross section as $b \bar{b} \rightarrow \phi$. In the partonic calculation, the bottom-quark mass is set to zero (except where it occurs in the Yukawa coupling), and all collinear divergences are absorbed into the parton density functions (PDFs) through mass factorization. Concerning the sub-process $g g \rightarrow \phi b \bar{b}$, there is a potential mismatch of this approach with the treatment of the bottomquark threshold in the parton densities. However, by comparing the massless with a massive calculation in this subchannel [7], such effects could be shown to be negligible w.r.t. the overall theoretical accuracy.

The current experimental analyses are based on a combination of results from both approaches through the so-called Santander-matching formula [8], where the 4FS and 5FS results- the former at next-to-leading order (NLO) $[9,10]$, the latter at next-to-NLO (NNLO) QCD [11]—enter with Higgs-mass dependent weights. For larger Higgs mass, the logarithms discussed above become more important, so the 
5FS is expected to provide the more reliable result, and thus receives a larger weight. This is indeed confirmed by approaches aiming at a theoretically better-founded matching of the underlying processes [12-14]. ${ }^{1}$

Due to the small value of the charm-quark mass $m_{c} \sim 1 \mathrm{GeV}$, a charm-initiated approach for the calculation of the total inclusive cross section, $c \bar{c} \rightarrow \phi$, is preferable over a 3 -flavor scheme (3FS) description (LO process $g g \rightarrow c \bar{c} \phi$ ) already for much smaller values of the Higgs-boson mass. It can be evaluated both in the 4FS and the 5FS, where in the latter case the bottom quark plays the role of a spectator. Since, as we will show below, interference effects involving the bottom and the charm Yukawa coupling are absent, the only technical difference in evaluating the 4FS and the 5FS result for $\sigma(c \bar{c} \rightarrow \phi)$ is a change of the PDF set. All results in this paper are obtained in the 5FS.

Analogous considerations apply to other quark-associated production modes. As we will show in this paper, the corresponding NNLO partonic cross sections differ only by an obvious overall factor, given by the ratio of the respective Yukawa couplings, as long as the dynamical quark masses (as opposed to the Yukawa couplings) are neglected. The latter condition is anyway necessary in a partonic formulation of these scattering processes.

We can therefore use the known partonic NNLO results for the process $b \bar{b} \rightarrow \phi[11]$, and translate them into hadronic cross sections for arbitrary initial-state quarks. This will be explained in more detail in the next section. Section 3 uses these results to determine the central renormalization and factorization scales for all heavy-quark initiated Higgs production processes, and provides theoretical predictions through NNLO. In addition, the impact of hard radiation is disentangled from the purely PDF-induced effects. Section 4 contains our conclusions.

\section{Calculation}

We denote by $Q^{\prime} \bar{Q} \phi$ the process for the associated production of a Higgs boson $\phi$ with a $Q^{\prime} \bar{Q}$ pair in the 5FS, whose LO Feynman diagram is given by Fig. 1. Depending on the specific flavors of $Q$ and $Q^{\prime}, \phi$ can be electrically neutral or charged. Within QCD, renormalization of the $Q^{\prime} \bar{Q} \phi$ coupling, and thus also its anomalous dimension, is independent of the quark flavors $Q$ and $Q^{\prime}$. Since we work in the massless-quark limit throughout this paper, the underlying theory is chirally symmetric, which means that all our results apply to scalar as well as pseudo-scalar Higgs bosons $\phi$ (see also Ref. [11]); scalar/pseudo-scalar interference terms vanish.

\footnotetext{
${ }^{1}$ For a comparison of differential distributions in $b \bar{b} \phi$ production based on the 4FS and the 5FS, see Ref. [15].
}

Fig. 1 LO Feynman diagram for $Q \bar{Q}^{\prime} \rightarrow \phi$, defining the process $p p \rightarrow Q^{\prime} \bar{Q} \phi$ in the $5 \mathrm{FS}$

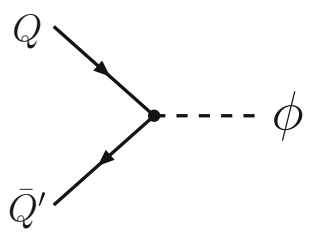

At NLO QCD, aside from the virtual corrections to the LO process, the real radiation processes $Q \bar{Q}^{\prime} \rightarrow g \phi, g Q \rightarrow Q^{\prime} \phi$, and $g \bar{Q}^{\prime} \rightarrow \bar{Q} \phi$ need to be taken into account in the calculation of the total cross section. Similarly, at NNLO QCD, there are the 2-loop virtual corrections to the LO process, and the 1loop virtual corrections to the NLO real-emissions processes. In addition, double-real emission processes occur. Those with two external gluons are: $Q \bar{Q}^{\prime} \rightarrow g g \phi, Q g \rightarrow Q g \phi$, $\bar{Q}^{\prime} g \rightarrow \bar{Q}^{\prime} g \phi, g g \rightarrow \bar{Q} Q^{\prime} \phi$. The squared amplitude composed of these processes contains only a single fermionic trace.

This may be different for processes with four external quarks. Their amplitudes are given by
A: $Q \bar{Q}^{\prime} \rightarrow q \bar{q} \phi$
B: $q \bar{q} \rightarrow Q^{\prime} \bar{Q} \phi$
$\mathrm{C}: Q q \rightarrow Q^{\prime} q \phi, \quad \bar{Q}^{\prime} q \rightarrow \bar{Q} q \phi, \quad Q \bar{q} \rightarrow Q^{\prime} \bar{q} \phi, \quad \bar{Q}^{\prime} \bar{q} \rightarrow$ $\bar{Q} \bar{q} \phi$

and correspond to the Feynman diagrams shown in Fig. 2. Here, $q$ denotes a quark of arbitrary flavor, and $\bar{q}$ the corresponding anti-quark. The square of each of these processes involves two fermionic traces, one of which contains both Higgs couplings.

Let us now look at potential interference terms. If $q \notin$ $\left\{Q, Q^{\prime}\right\}$, the initial and final states of A, B, and C are different, and they obviously cannot interfere. If $q \in\left\{Q, Q^{\prime}\right\}$, there are $\mathrm{AC}$ and $\mathrm{BC}$ interference terms, which involve a single fermionic trace.

All contributions above are independent of the specific quark flavors $Q$ and $Q^{\prime}$. For $Q=Q^{\prime}=q$, however, it seems that also $A$ and $B$ interfere with each other, leading to a term with two fermionic traces, each of which contains one Higgs coupling. However, in the limit of zero quark masses, the traces are over an odd number of Dirac matrices and vanish.

In conclusion, aside from an overall constant Yukawa factor, ${ }^{2}$ the NNLO partonic cross section for the process $Q^{\prime} \bar{Q} \phi$ is independent of the quark flavors $Q$ and $Q^{\prime}$, as long as quark masses are neglected. Along the same lines, one observes that, for $Q \neq Q^{\prime}$, any interference terms between $Q \bar{Q}$ - and $Q^{\prime} \bar{Q}^{\prime}$-initiated Higgs production vanishes through NNLO.

Let us remark that in the analogous case of Drell-Yan production, i.e. $\phi=V \in\{W, Z\}$, the $\mathrm{AB}$ interference term,

\footnotetext{
${ }^{2}$ Recall that the anomalous dimension of the $Q^{\prime} \bar{Q} \phi$ vertex is independent of $Q$ and $Q^{\prime}$; see above.
} 
Fig. 2 NNLO contributions to the $Q^{\prime} \bar{Q} \phi$ process which involve four external quarks. $\mathbf{c}$ is a representative for three more diagrams which are obtained by replacing $q \rightarrow \bar{q}$, or $\left(Q, Q^{\prime}\right) \rightarrow\left(\bar{Q}^{\prime}, \bar{Q}\right)$, or both

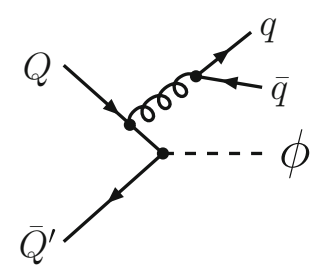

(A)

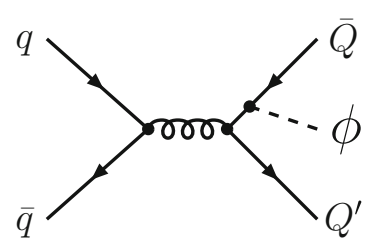

(B)

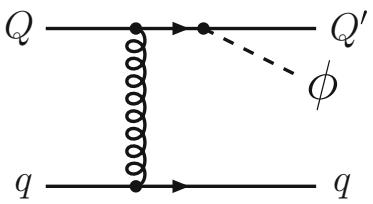

(C)

which exists only for $Z$-production, is not zero. The doublequark emission corrections for $W$-production are therefore different from those of $Z$-production $[16,17]$. $^{3}$

It follows that the hadronic $Q^{\prime} \bar{Q} \phi$ cross section for the collision of hadrons $h_{1}$ and $h_{2}$ can be obtained by simply convolving the $5 \mathrm{FS}$ partonic cross section for $b \bar{b} \phi$ production with the appropriate PDFs. For example, we may define

$\sigma_{b \bar{b}}\left(f, f^{\prime}\right)=\left[f_{1} \otimes f_{2}^{\prime}+f_{1}^{\prime} \otimes f_{2}\right] \otimes \hat{\sigma}_{b \bar{b}}$,

where $\hat{\sigma}_{b \bar{b}}=\hat{\sigma}_{b \bar{b}}\left(m_{H}^{2} / \hat{s}\right)$ is the partonic cross section for the SM process $b \bar{b} \rightarrow H+X$, which can be found in Ref. [11], $\hat{s}$ is the partonic center-of-mass energy, and $\otimes$ denotes the convolution

$(f \otimes g)(x)=\int_{0}^{1} \mathrm{~d} x_{1} \int_{0}^{1} \mathrm{~d} x_{2} f\left(x_{1}\right) g\left(x_{2}\right) \delta\left(x-x_{1} x_{2}\right)$.

Furthermore, $f_{j}(x)$ and $f_{j}^{\prime}(x)$ are the parton densities (multiplied by $x$ ) in the hadron $h_{j}$, with $f, f^{\prime} \in\{q, \bar{q}, g\}$ and $q \in\{d, u, s, c, b\}$. The component of the hadronic $Q^{\prime} \bar{Q} \phi$ cross section which is induced by the partonic $Q \bar{Q}^{\prime}$ initial state can then be written as

$\sigma\left(Q \bar{Q}^{\prime} \rightarrow \phi+X\right)=\beta_{Q Q^{\prime}} \sigma_{b \bar{b}}\left(Q, \bar{Q}^{\prime}\right)$

where $\beta_{Q Q^{\prime}}$ is the squared ratio of the $Q \bar{Q}^{\prime} \phi$ and the SM $b \bar{b} H$ coupling. In particular, we have $\sigma(b \bar{b} \rightarrow H+X)=$ $\sigma_{b \bar{b}}(b, \bar{b})$.

Similarly, we can define

$$
\begin{aligned}
\sigma_{b g}\left(f, f^{\prime}\right) & =\left[\left(f_{1}+f_{1}^{\prime}\right) \otimes g_{2}+g_{1} \otimes\left(f_{2}+f_{2}^{\prime}\right)\right] \otimes \hat{\sigma}_{b g}, \\
\sigma_{b b}\left(f, f^{\prime}\right) & =\left[f_{1} \otimes f_{2}+f_{1}^{\prime} \otimes f_{2}^{\prime}\right] \otimes \hat{\sigma}_{b b}, \\
\sigma_{b q}\left(f, f^{\prime}\right) & =\left[\left(f_{1}+f_{1}^{\prime}\right) \otimes \Sigma_{2}+\Sigma_{1} \otimes\left(f_{2}+f_{2}^{\prime}\right)\right] \otimes \hat{\sigma}_{b q}, \\
\sigma_{g g} & =g_{1} \otimes g_{2} \otimes \hat{\sigma}_{g g}, \\
\sigma_{q \bar{q}} & =\sum_{q}\left(q_{1} \otimes \bar{q}_{2}+q_{2} \otimes \bar{q}_{1}\right) \otimes \hat{\sigma}_{q \bar{q}},
\end{aligned}
$$

\footnotetext{
${ }^{3}$ This effect adds to the difference between $W$ - and $Z$-production arising from other contributions; see Refs. $[16,17]$ for more details. Note that the same discussion also applies to the Higgs-Strahlung process, $p p \rightarrow V H$. In this case, however, there is a much more important difference between $V=W$ and $V=Z$ arising from the gluon induced $g g \rightarrow H Z$ process [18-21].
}

where

$\Sigma_{i}=\sum_{q}\left(q_{i}+\bar{q}_{i}\right)-f_{i}-f_{i}^{\prime}$,

and the sum runs over all quark flavors $q$. The partonic cross sections $\hat{\sigma}_{i j}$ on the right hand side are $i j$-initiated components of the partonic SM $b \bar{b} H$ cross section; explicit expressions can be found in Ref. [11].

In this way, we can calculate

$$
\begin{aligned}
& \sigma(Q g \rightarrow \phi+X)+\sigma\left(\bar{Q}^{\prime} g \rightarrow \phi+X\right)=\beta_{Q Q^{\prime}} \sigma_{b g}\left(Q, \bar{Q}^{\prime}\right), \\
& \sigma(Q Q \rightarrow \phi+X)+\sigma\left(\bar{Q}^{\prime} \bar{Q}^{\prime} \rightarrow \phi+X\right)=\beta_{Q Q^{\prime}} \sigma_{b b}\left(Q, \bar{Q}^{\prime}\right), \\
& \sigma(Q q \rightarrow \phi+X)+\sigma\left(\bar{Q}^{\prime} q \rightarrow \phi+X\right)=\beta_{Q Q^{\prime}} \sigma_{b q}\left(Q, \bar{Q}^{\prime}\right), \\
& \sigma(g g \rightarrow \phi+X)=\beta_{Q Q^{\prime}} \sigma_{g g}, \\
& \sigma(q \bar{q} \rightarrow \phi+X)=\beta_{Q Q^{\prime}} \sigma_{q \bar{q}},
\end{aligned}
$$

where $q$ may be any (anti-)quark except $Q$ or $\bar{Q}^{\prime}$. The total inclusive hadronic cross section is then given by the sum of all the terms in Eqs. (1) and (6).

The implementation of this result in bbh@nnlo[11] (which is now part of SusHi [22]) is straightforward and will be publicly available in the next version of SusHi. ${ }^{4}$

\section{Numerical results}

\subsection{Determination of the central scales}

As a reference, the upper two plots of Fig. 3 show the first three perturbative orders for the $b \bar{b} \phi$ cross section for $m_{\phi}=125 \mathrm{GeV}$ and $\beta_{b b}=1$ as a function of the factorization scale $\mu_{\mathrm{F}}$ (left), and the renormalization scale $\mu_{\mathrm{R}}$ (right). These results are well-known [11]; they corroborate the choice $\left(\hat{\mu}_{\mathrm{R}}, \hat{\mu}_{\mathrm{F}}\right)=(1,1 / 4)$ as the central values for the scales [23-25], where we have introduced the normalized scales

$\hat{\mu}_{\mathrm{R}} \equiv \mu_{\mathrm{R}} / m_{\phi}, \quad \hat{\mu}_{\mathrm{F}} \equiv \mu_{\mathrm{F}} / m_{\phi}$

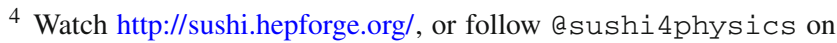
Twitter.
} 

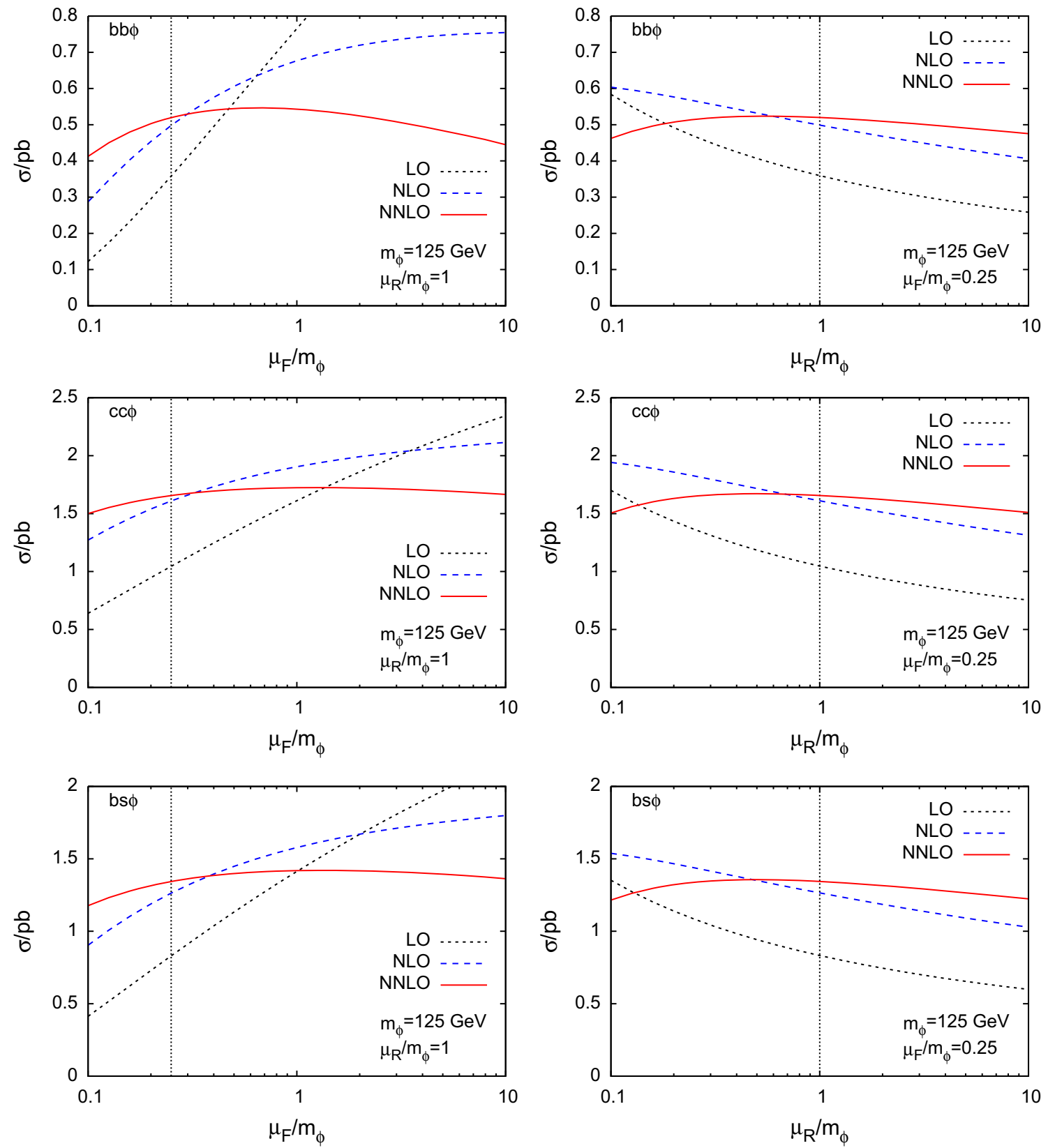

Fig. 3 LO (black dots), NLO (blue dashes), and NNLO result (solid red) for the total cross sections of the processes $b \bar{b} \phi, c \bar{c} \phi$, and $b \bar{s} \phi$ (top to bottom) at $m_{\phi}=125 \mathrm{GeV}$. Left column $\mu_{\mathrm{F}}$-dependence for $\mu_{\mathrm{R}}=m_{\phi}$ : right column $\mu_{\mathrm{R}}$-dependence for $\mu_{\mathrm{F}}=m_{\phi} / 4$. The vertical dotted lines

at $\hat{\mu}_{\mathrm{F}}=1 / 4($ left $)$ and $\hat{\mu}_{\mathrm{R}}=1($ right $)$ are introduced to guide the eye. At $\mathrm{N}^{n} \mathrm{LO}$ order, the corresponding central MSTW2008 set and its associated value of $\alpha_{s}\left(M_{Z}\right)$ has been used; $\alpha_{s}\left(M_{Z}\right)$ and $m_{b}\left(m_{b}\right)=4.18 \mathrm{GeV}$ have been evolved to $\mu_{\mathrm{R}}$ at $(n+1)$-loop order

We may formalize the justification of this choice by considering the variation $\Delta_{\mathrm{F}}$ of the NNLO hadronic cross section $\sigma$ within the interval $\hat{\mu}_{\mathrm{R}} \in[1 / 10,10]$, while fixing $\hat{\mu}_{\mathrm{F}}$ :

$\Delta_{\mathrm{F}}=\left.2 \frac{\max \sigma-\min \sigma}{\max \sigma+\min \sigma}\right|_{\hat{\mu}_{\mathrm{F}}}$.

The central factorization scale $\hat{\mu}_{\mathrm{F}}^{(0)}$ can then be defined as the value of $\hat{\mu}_{\mathrm{F}}$ where $\Delta_{\mathrm{F}}$ is minimal. The analogous procedure

(with $\mathrm{R} \leftrightarrow \mathrm{F}$ ) can be used to define the central renormalization scale $\hat{\mu}_{\mathrm{R}}^{(0)}$.

We performed this study for all heavy-quark initiated processes by calculating $\sigma$ on an equidistant $21 \times 21$ logarithmic grid in the $\left(\hat{\mu}_{\mathrm{R}}, \hat{\mu}_{\mathrm{F}}\right)$ plane, i.e., using the values $\hat{\mu}_{\mathrm{R}}, \hat{\mu}_{\mathrm{F}} \in\left\{10^{n / 10}, n=-10,-9, \ldots, 9,10\right\}$. When quoting numbers, we will round these values to two significant digits; e.g., we will refer to $\hat{\mu}_{\mathrm{F}}=10^{-3 / 5}=0.2512 \ldots$ simply as $\hat{\mu}_{\mathrm{F}}=0.25$, or $\hat{\mu}_{\mathrm{F}}=1 / 4$ for that matter. 

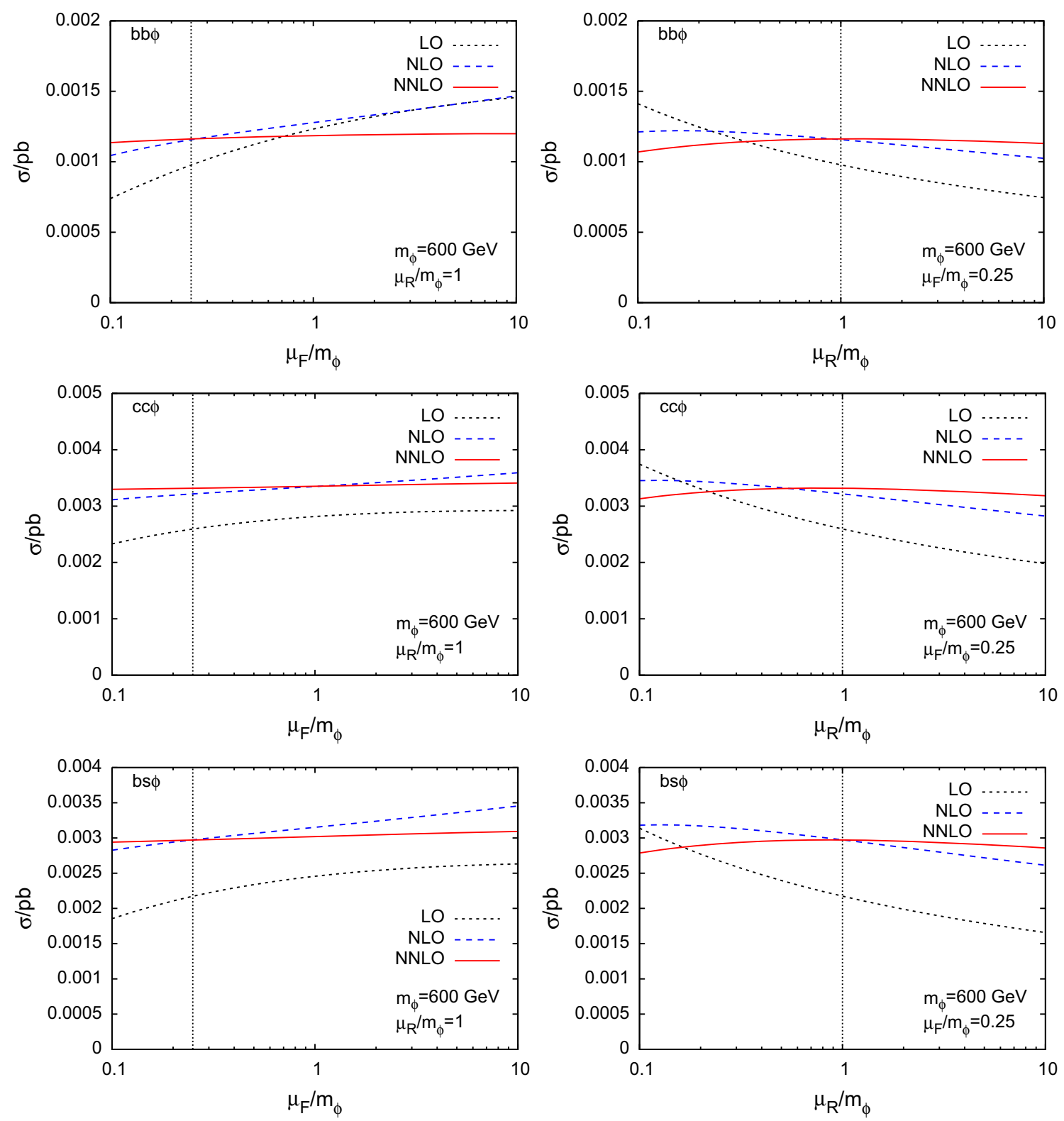

Fig. 4 Same as Fig. 3, but for $m_{\phi}=600 \mathrm{GeV}$

For $m_{\phi}=125 \mathrm{GeV}$, we find $\hat{\mu}_{\mathrm{F}}^{(0)}=1 / 4$ in this way, independent of the quark flavors $Q$ and $Q^{\prime}$. This is an interesting observation, because this value has been derived specifically for $Q=Q^{\prime}=b$ using kinematical considerations [23-25]; the fact that all other quark-initiated processes seem to favor the same $\hat{\mu}_{\mathrm{F}}^{(0)}$ is not at all obvious from these discussions. Following the above procedure, the central renormalization scale turns out to be $\hat{\mu}_{\mathrm{R}}^{(0)}=0.79$ for the $b \bar{b} \phi$ process, while for the other quark flavors we find $\hat{\mu}_{\mathrm{R}}^{(0)}=0.63$.

For $m_{\phi}=600 \mathrm{GeV}$, all processes favor an even smaller value of the factorization scale, namely $\hat{\mu}_{\mathrm{F}}^{(0)}=0.16$. Also the central renormalization scale comes out smaller: we find $\hat{\mu}_{\mathrm{R}}^{(0)}=0.63$ for $b \bar{b} \phi, \hat{\mu}_{\mathrm{R}}^{(0)}=0.5$ for $c \bar{c} \phi$ and $b \bar{c} \phi^{+}$, and $\hat{\mu}_{\mathrm{R}}^{(0)}=0.4$ for $b \bar{s} \phi$ and $c \bar{s} \phi^{-}$.

However, in all cases, the minima are sufficiently shallow to justify also the choice $\left(\hat{\mu}_{\mathrm{R}}^{(0)}, \hat{\mu}_{\mathrm{F}}^{(0)}\right)=(1,1 / 4)$. Exemplary plots for the $b \bar{b} \phi, c \bar{c} \phi$, and $b \bar{s} \phi$ processes are shown in Fig. 3 for $m_{\phi}=125 \mathrm{GeV}$, and in Fig. 4 for $m_{\phi}=$ $600 \mathrm{GeV}$. All cross sections correspond to $\beta_{Q Q^{\prime}}=1$, i.e., the Yukawa coupling is assumed identical to the one for SM $b \bar{b} H$ production. For the $\mathrm{N}^{n} \mathrm{LO}$ curve, it is evaluated from $m_{b}\left(m_{b}\right)=4.18 \mathrm{GeV}$ by $(n+1)$-loop evolution with $n_{f}=5$ 
active flavors to ${ }^{5} m_{b}\left(\mu_{\mathrm{R}}\right) \equiv m_{b}^{(5)}\left(\mu_{\mathrm{R}}\right)$. Thus, in order to derive the $c \bar{c} \phi$ cross section within the SM, for example, the plots in the second rows of Figs. 3 and 4 should be scaled by $\beta_{c c}=\left(m_{c}^{(5)}\left(m_{b}\right) / m_{b}^{(5)}\left(m_{b}\right)\right)^{2} \approx 0.049$, where we have used 4-loop running to determine $m_{c}^{(5)}\left(m_{b}\right)=$ $0.926 \mathrm{GeV}$ from $m_{c}^{(4)}(3 \mathrm{GeV})=0.986 \mathrm{GeV}$ [26]. In the $\mathrm{SM}$, the $c \bar{c} \phi$ cross section is therefore about 6-7 times smaller than the $b \bar{b} \phi$ cross section. All plots have been produced with the MSTW2008 PDF sets [27] as implemented in the LHAPDF library [28,29], and the associated value of $\alpha_{s}\left(M_{Z}\right)=0.139 / 0.120 / 0.117$ at LO/NLO/NNLO.

Recall that the role of the central values is to determine the position of a "reasonable" interval for $\hat{\mu}_{\mathrm{F}}$ and $\hat{\mu}_{\mathrm{R}}$; the variation of the cross section within this interval should then give a clue of the associated theoretical error induced by missing higher-order effects. Due to the unphysical nature of the renormalization and factorization scale, any procedure to "determine" their central values is formally arbitrary, though not necessarily sensible. The fact that, at $\left(\hat{\mu}_{\mathrm{R}}, \hat{\mu}_{\mathrm{F}}\right)=\left(\hat{\mu}_{\mathrm{R}}^{(0)}, \hat{\mu}_{\mathrm{F}}^{(0)}\right)$, the NNLO corrections are significantly smaller than the NLO ones in all cases studied here (see Figs. 3, 4), confirms that the procedure defined above is indeed sensible. Other observations concerning the choice of the central scale in the case of the $Q^{\prime} \bar{Q} \phi$ processes will be recalled in Sect. 3.4.

\subsection{Cross sections and uncertainties}

Numerical values for the cross sections at the LHC with $13 \mathrm{TeV}$ are shown in the form $\langle\sigma\rangle \pm \Delta_{\mu} \pm \Delta_{\mathrm{PDF}}$ in Table 1, where $\Delta_{\mu}$ and $\Delta_{\mathrm{PDF}}$ are the scale and PDF uncertainty, respectively, to be defined below. Again, for all processes, we assumed the Yukawa coupling to be equal to the $\mathrm{SM} b \bar{b} \phi$ coupling. For these numbers, we evolved $m_{b}\left(m_{b}\right)=4.18 \mathrm{GeV}$ to $m_{b}\left(m_{\phi}\right)$ at 4-loop level, and subsequently $m_{b}\left(m_{\phi}\right)$ to $m_{b}\left(\mu_{\mathrm{R}}\right)$ at 3-loop level. The numerical difference to the single-step 4-loop evolution from $m_{b}\left(m_{b}\right)$ to $m_{b}\left(\mu_{\mathrm{R}}\right)$ as used in all the plots of this paper is negligible.

The MMHT2014 PDF set is employed for the convolution of the partonic cross section. ${ }^{6}$ At NNLO, it uses $\alpha_{s}\left(M_{Z}\right)=0.118$, and an on-shell bottom-quark mass of $m_{b, \mathrm{PDF}}=m_{b}^{\mathrm{OS}}=4.75 \mathrm{GeV}$, which is very close to $4.78 \mathrm{GeV}$, the value obtained by 2-loop conversion from $m_{b}\left(m_{b}\right)=$ $4.18 \mathrm{GeV}$ to the on-shell scheme (1-loop conversion gives $4.56 \mathrm{GeV}, 3-$, and 4-loop conversions both lead to $4.93 \mathrm{GeV}$ ).

\footnotetext{
$\overline{5 \text { The notation }} m_{q}^{\left(n_{f}\right)}$ indicates that $m_{q}$ is renormalized in $n_{f}$-flavor QCD.

${ }^{6}$ Since this section aims at presenting the most up-to-date cross section predictions, we make use of latest-generation PDF sets here. The reason for using older sets in Sect. 3.1 is a technical one; it has no effect on the values for the central scales obtained there.
}

Table 1 Cross sections for the processes $Q^{\prime} \bar{Q} \phi$ at $13 \mathrm{TeV}$. The $Q^{\prime} \bar{Q} \phi$ coupling is assumed equal to the SM $b \bar{b} H$ coupling in all cases. The cross sections hold for both scalar and pseudo-scalar Higgs bosons. The first uncertainty is due to scale variation, the second one denotes the PDF error (see main text for more details)

\begin{tabular}{lll}
\hline$M_{\phi} / \mathrm{GeV}$ & $Q^{\prime} \bar{Q} \phi$ & $\sigma / \beta_{Q Q^{\prime}} / \mathrm{pb}$ \\
\hline 125 & $b \bar{b} \phi$ & $(5.23 \pm 0.58 \pm 0.11) \cdot 10^{-1}$ \\
& $c \bar{c} \phi$ & $(1.64 \pm 0.08 \pm 0.04) \cdot 10^{0}$ \\
& $b \bar{c} \phi^{+}$ & $(9.35 \pm 0.76 \pm 0.23) \cdot 10^{-1}$ \\
& $b \bar{s} \phi$ & $(1.39 \pm 0.09 \pm 0.12) \cdot 10^{0}$ \\
& $c \bar{s} \phi^{-}$ & $(2.44 \pm 0.10 \pm 0.21) \cdot 10^{0}$ \\
& $b \bar{b} \phi$ & $(1.19 \pm 0.02 \pm 0.05) \cdot 10^{-3}$ \\
& $c \bar{c} \phi$ & $(3.38 \pm 0.02 \pm 0.15) \cdot 10^{-3}$ \\
& $b \bar{c} \phi^{+}$ & $(2.02 \pm 0.03 \pm 0.08) \cdot 10^{-3}$ \\
& $b \bar{s} \phi$ & $(2.97 \pm 0.03 \pm 0.28) \cdot 10^{-3}$ \\
& $c \bar{s} \phi^{-}$ & $(4.95 \pm 0.03 \pm 0.46) \cdot 10^{-3}$ \\
& $b \bar{b} \phi$ & $(4.04 \pm 0.06 \pm 0.19) \cdot 10^{-4}$ \\
& $c \bar{c} \phi$ & $(1.15 \pm 0.01 \pm 0.06) \cdot 10^{-3}$ \\
& $b \bar{c} \phi^{+}$ & $(6.87 \pm 0.06 \pm 0.34) \cdot 10^{-4}$ \\
& $b \bar{s} \phi$ & $(1.01 \pm 0.01 \pm 0.10) \cdot 10^{-3}$ \\
& $c \bar{s} \phi^{-}$ & $(1.70 \pm 0.01 \pm 0.17) \cdot 10^{-3}$ \\
\hline
\end{tabular}

The dependence of the cross sections on the heavy-quark masses will be discussed in more detail below.

We evaluated the cross section for the seven pairs of scales $^{7}$

$$
\begin{array}{r}
\left(\hat{\mu}_{\mathrm{R}}, \hat{\mu}_{\mathrm{F}}\right)=\{(1 / 2,1 / 8),(1 / 2,1 / 4),(1,1 / 8),(1,1 / 4), \\
(1,1 / 2),(2,1 / 4),(2,1 / 2)\},
\end{array}
$$

using the central NNLO PDF set of MMHT2014 [30]. The corresponding maximal/minimal values of the cross section, $\sigma_{\mu}^{\max / \min }$, then determine the scale error interval as $\Delta_{\mu}=\left(\sigma_{\mu}^{\max }-\sigma_{\mu}^{\min }\right) / 2$ quoted in Table 1. The PDF uncertainty $\Delta_{\mathrm{PDF}}$ is determined from the 25 eigenvector sets of MMHT2014 while setting $\left(\hat{\mu}_{\mathrm{R}}, \hat{\mu}_{\mathrm{F}}\right)=(1,1 / 4)$; the central value $\langle\sigma\rangle$ is the mid-point of the PDF error interval.

We checked that we obtain comparable results when using the CT14 PDF set [31], while the default NNPDF 3 . 0 set [32] typically leads to larger $b \bar{b} \phi$ and $b \bar{c} \phi^{+}$cross sections (15\% and $11 \%$ at $m_{\phi}=125 \mathrm{GeV}$, respectively), which is most likely due to the significantly smaller bottom-quark mass assumed in that set $\left(\left.m_{b}\right|_{\text {NNPDF } 3.0}=4.18 \mathrm{GeV}\right)$. This motivates a study of the sensitivity of the results to the heavyquark masses $m_{b, \mathrm{PDF}}, m_{c, \mathrm{PDF}}$ used in the PDF fits. To that

\footnotetext{
7 In Table 1, we do not use the grid values, but actually set $1 / 4=$ $0.25000 \ldots$, etc.
} 

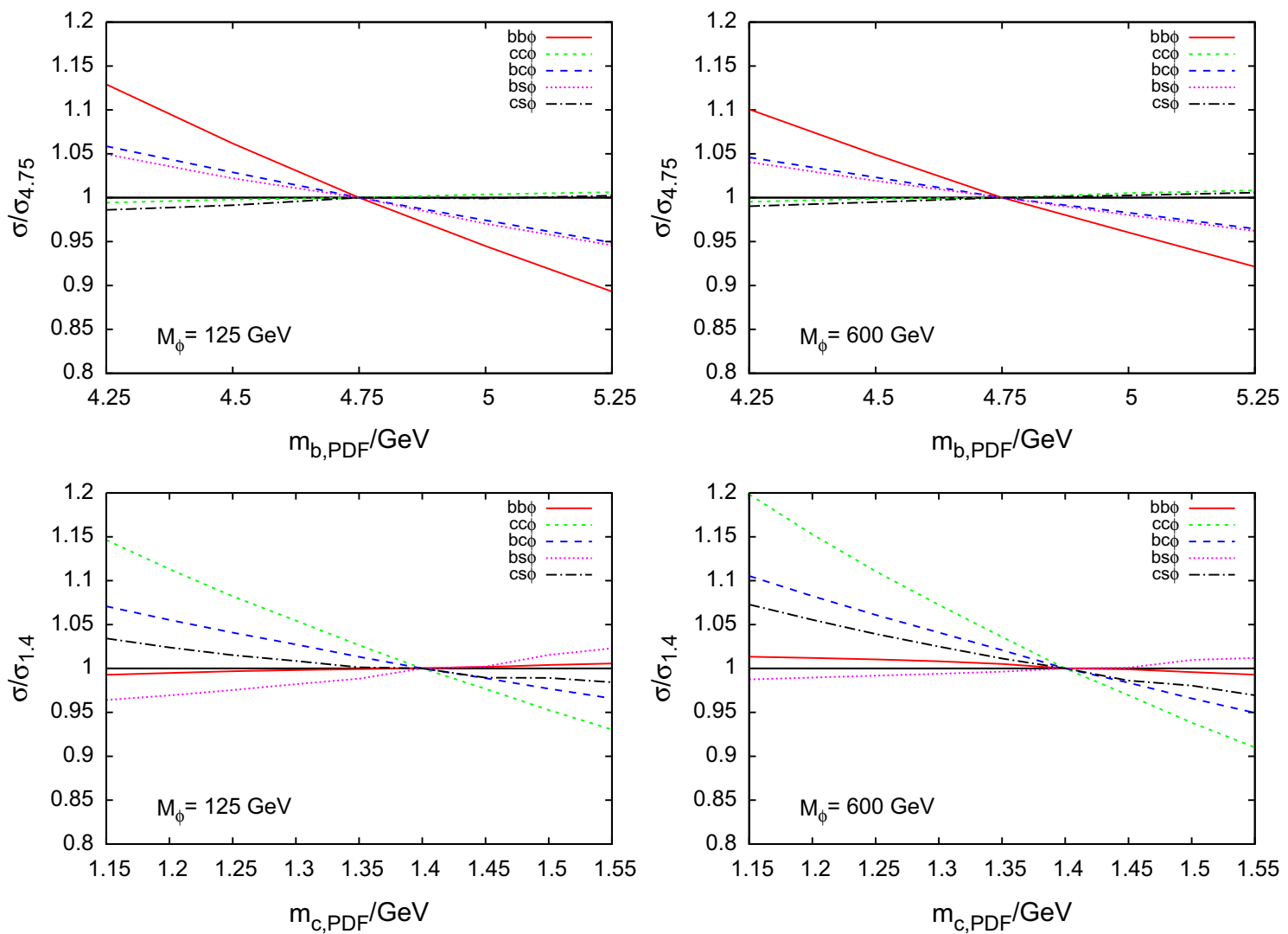

Fig. 5 Dependence of the hadronic cross section on the heavy-quark mass value $m_{q}$,PDF used in the PDF fit (curves for MMHT2014). Upper row $q=b$; lower row $q=c$. Left $_{\phi}=125 \mathrm{GeV}$; right $m_{\phi}=600 \mathrm{GeV}$. Normalization is to the default PDF set with $\left(m_{b, \mathrm{PDF}}, m_{c, \mathrm{PDF}}\right)=(4.75,1.4) \mathrm{GeV}$

aim, we use the mbrange_nf5 and mcrange_nf5 versions of MMHT2 014 , whose member sets correspond to different values of the bottom- and charm-quark masses. Figure 5 shows the $Q^{\prime} \bar{Q} \phi$ cross sections (interpolated between the discrete quark-mass values) for each of these sets. All curves are evaluated at $\left(\hat{\mu}_{\mathrm{R}}, \hat{\mu}_{\mathrm{F}}\right)=(1,1 / 4)$ for fixed Yukawa coupling (determined from $m_{b}\left(m_{b}\right)=4.18 \mathrm{GeV}$ as described above), and they are normalized to the one with the default PDF quark-mass values $\left(m_{b, \mathrm{PDF}}, m_{c, \mathrm{PDF}}\right)=$ $(4.75,1.4) \mathrm{GeV}$. We observe that the cross sections and the PDF quark masses are anti-correlated, and that a change of $m_{b, \mathrm{PDF}}$ by $1 \%$ changes the $b \bar{b} \phi$ cross section by about $1.5 \%$ at $m_{\phi}=125 \mathrm{GeV}$, and a little less at $m_{\phi}=600 \mathrm{GeV}$; the $b \bar{c} \phi^{+}$and $b \bar{s} \phi$ cross sections change by about $0.7 \%$. On the other hand, the $c \bar{c} \phi$ cross section for $m_{\phi}=125 \mathrm{GeV}$ changes by about $0.9 \%$ when varying $m_{c \text {, PDF }}$ by $1 \%$, and a little more at $m_{\phi}=600 \mathrm{GeV}$. Note that such a variation is largely compensated by a corresponding change of the quark mass in the Yukawa coupling (see Ref. [33]).

Note that, for the $b \bar{b} \phi$ process, a much more thorough account of the quark-mass effects has been obtained by a consistent matching between the various energy regimes involved in this process $[13,14]$. A similar analysis could be performed for the general $Q^{\prime} \bar{Q} \phi$ processes discussed here; however, we expect the corresponding effects to be much smaller than other theoretical and expected experimental uncertainties for these processes.

\subsection{Effects due to the hard process}

At LO, the partonic $Q^{\prime} \bar{Q} \phi$ cross section is

$\hat{\sigma}(x) \sim \delta(1-x), \quad x=m_{\phi}^{2} / \hat{s}$,

and thus the LO hadronic cross section for $Q^{\prime} \bar{Q} \phi$ production is proportional to the $\mathrm{LO} b \bar{b} \phi$ cross section,

$\sigma^{\mathrm{LO}}\left(Q^{\prime} \bar{Q} \phi\right)=\frac{\beta_{Q Q^{\prime}} \mathcal{E}\left(Q^{\prime}, \bar{Q}\right)}{\mathcal{E}(b, \bar{b})} \sigma^{\mathrm{LO}}(b \bar{b} \phi)$,

where

$\mathcal{E}\left(f, f^{\prime}\right) \equiv f_{1} \otimes f_{2}^{\prime}+f_{1}^{\prime} \otimes f_{2}$

is the $f f^{\prime}$ parton luminosity in $h_{1} h_{2}$ collisions. To a first approximation, one might be tempted to apply the rescaling of Eq. (11) also at higher orders. In order to see to what extent such an approximation is valid, let us study the double ratio 

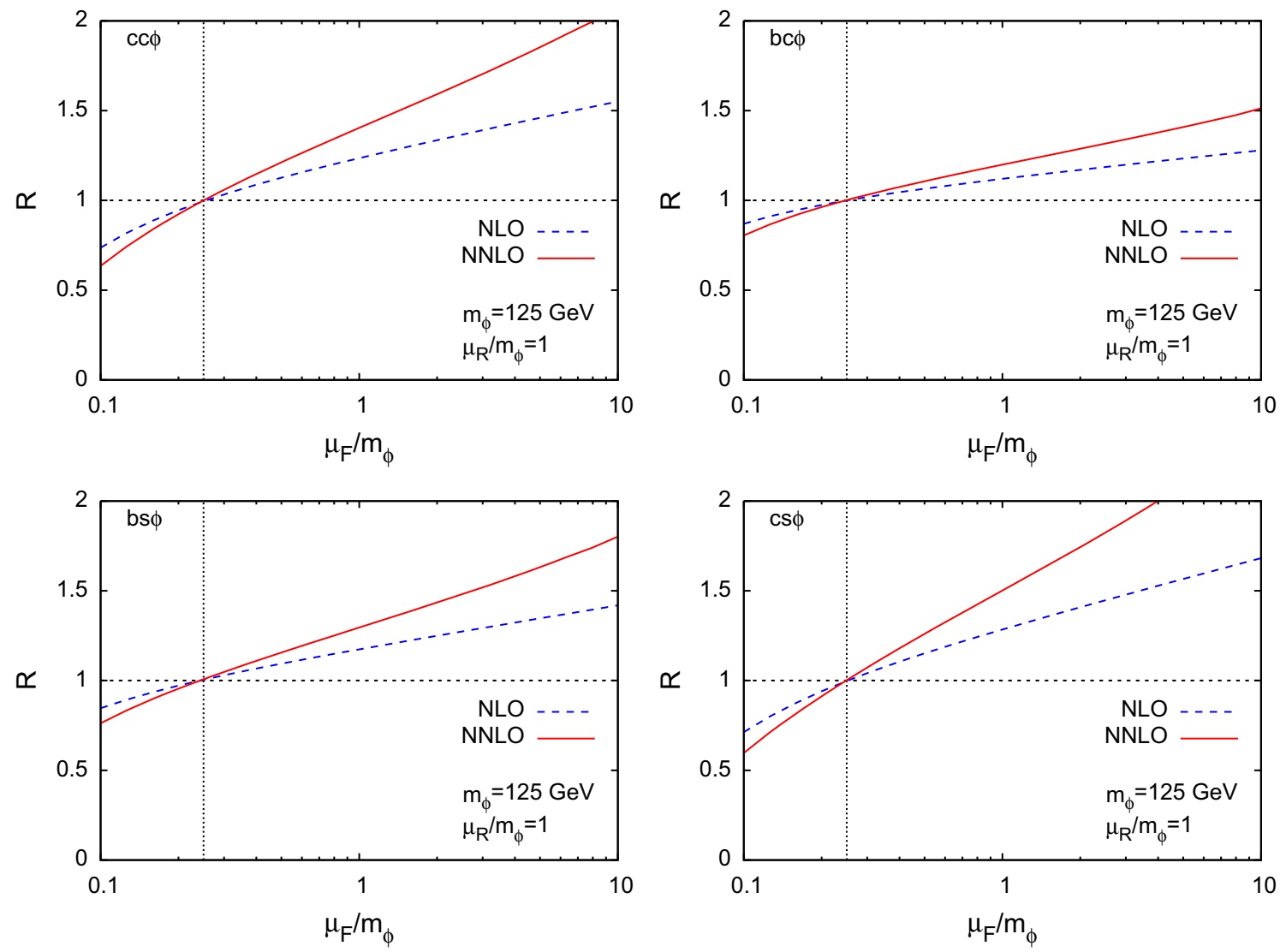

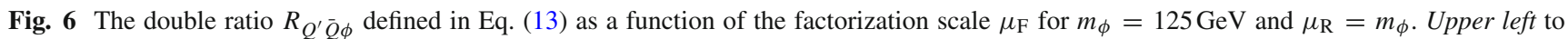
lower right: $Q^{\prime} \bar{Q} \phi=c \bar{c} \phi, b \bar{c} \phi^{+}, b \bar{s} \phi, c \bar{s} \phi^{-}$. Dashed NLO; Solid NNLO

$R_{Q^{\prime} \bar{Q} \phi}=\frac{\sigma\left(Q^{\prime} \bar{Q} \phi\right) / \sigma(b \bar{b} \phi)}{\beta_{Q Q^{\prime}} \mathcal{E}\left(Q^{\prime}, \bar{Q}\right) / \mathcal{E}(b, \bar{b})}$

at NLO and NNLO QCD, where at each order it is understood that we use the appropriate set of PDFs, e.g., MSTW2 008 nlo at NLO and MSTW2008nnlo at NNLO.

Any deviation from $R_{Q^{\prime}} \bar{Q} \phi=1$ is due to the hard scattering process. These effects depend on the choice of $\hat{\mu}_{\mathrm{R}}$ and $\hat{\mu}_{\mathrm{F}}$. Figure 6 shows the $\hat{\mu}_{\mathrm{F}}$ dependence of $R_{Q^{\prime} \bar{Q} \phi}$ at $\hat{\mu}_{\mathrm{R}}=1$ for $m_{\phi}=125 \mathrm{GeV}$; the effects typically decrease/increase toward larger/smaller $\hat{\mu}_{\mathrm{R}}$. Interestingly, one observes that also here the scale $\hat{\mu}_{\mathrm{F}}=1 / 4$ plays a special role: in all cases, the hard-scattering effects become minimal around this value. Moreover, we have checked that this observation is virtually independent of $\hat{\mu}_{\mathrm{R}}$ (at least within $\hat{\mu}_{\mathrm{R}} \in[1 / 10,10]$ ). For $m_{\phi}=600 \mathrm{GeV}$, one observes an analogous behavior, albeit again at slightly lower $\hat{\mu}_{\mathrm{F}}$; see Fig. 7 .

\subsection{Remarks on the central scale}

Despite the fact that the "determination" of a central scale is not an unambiguous concept, it may be worth recalling the special role of this quantity for the $Q^{\prime} \bar{Q} \phi$ processes. Let us focus on the case $m_{\phi}=125 \mathrm{GeV}$ for the sake of simplic- ity. On the one hand, our studies of Sect.3.1 have singled out the values $\left(\hat{\mu}_{\mathrm{R}}, \hat{\mu}_{\mathrm{F}}\right)=(1,1 / 4)$ as preferable from a perturbative point of view; see also Ref. [11]. As noted above, this is compatible with kinematic considerations based on the behavior of the bottom-quark parton densities [23-25]. In addition, it turns out that, for the $b \bar{b} \phi$ process, the LO perturbative predictions within the 4FS and the 5FS agree at the 5-10\% level for this choice of scales, while they differ by more than a factor of four for $\left(\hat{\mu}_{\mathrm{R}}, \hat{\mu}_{\mathrm{F}}\right)=(1,1)$, for example [34]. Furthermore, it was found that the total cross section is almost completely exhausted by the partonic $b \bar{b}$ channel at $\left(\hat{\mu}_{\mathrm{R}}, \hat{\mu}_{\mathrm{F}}\right)=(1,1 / 4)$, while all other channels are very small [35]. Specifically, for $m_{\phi}=125 \mathrm{GeV}$, one finds

$\sigma(b \bar{b} \phi)=\sigma(b \bar{b} \phi)[1.04-0.06+0.02]$ at

$\left(\hat{\mu}_{\mathrm{R}}, \hat{\mu}_{\mathrm{F}}\right)=(1,1 / 4)$,

where the first number in square brackets denotes the $b \bar{b}$, the second the $(b+\bar{b}) g$, and the third the sum of the remaining channels. For $\left(\hat{\mu}_{\mathrm{R}}, \hat{\mu}_{\mathrm{F}}\right)=(1,1)$, on the other hand, we find a large cancellation between the $b \bar{b}$ and the $(b+\bar{b}) g$ channel:

$\sigma(b \bar{b} \phi)=\sigma(b \bar{b} \phi)[2.39-1.56+0.17]$ at

$\left(\hat{\mu}_{\mathrm{R}}, \hat{\mu}_{\mathrm{F}}\right)=(1,1)$. 

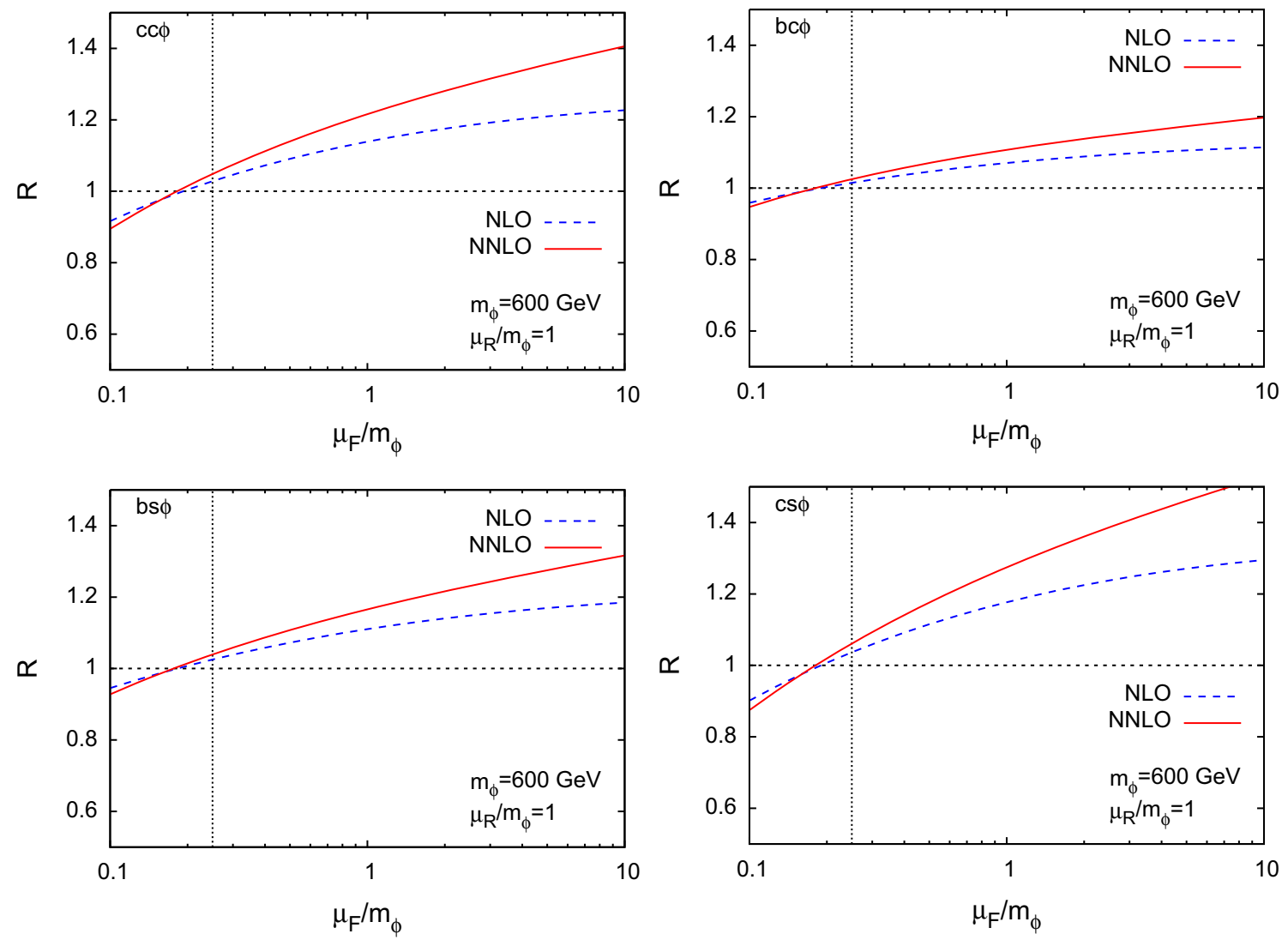

Fig. 7 Same as Fig. 6, but for $m_{\phi}=600 \mathrm{GeV}$

That latter observation seems closely related to the one of Sect. 3.3 which shows that the differences between different initial-state partons due to genuinely hard effects practically vanish at $\left(\hat{\mu}_{\mathrm{R}}, \hat{\mu}_{\mathrm{F}}\right)=(1,1 / 4)$. For the sake of completeness, let us note that analogous observations hold at larger Higgs masses, albeit at slightly different values for the central scales.

The author is not aware of any solid theoretical arguments that would explain why all of these observations occur at this one particular choice for the unphysical scales; one may simply characterize them as a mere curiosity. On the other hand, if there is an explanation, it would certainly be useful for the reduction of the theoretical uncertainty in perturbative calculations.

\section{Conclusions}

Cross sections for the production of neutral and charged scalar and pseudo-scalar particles via quark annihilation have been calculated through NNLO QCD. The results should be useful for studying models with an extended Higgs sector at hadron colliders. Explicit predictions for cross sections in exemplary cases of a SM-like and a heavy Higgs boson have been provided. For more general analyses, the next version of the public program SusHi [22] will provide easy access to these cross sections.

As a final remark, we note that the considerations of Sect. 2 can be extended to higher orders of perturbation theory and are not restricted to the total inclusive cross section. The $\mathrm{N}^{3} \mathrm{LO}$ results of Refs. [36,37] as well as the differential results of Refs. [38-41] can therefore be generalized to $Q^{\prime} \bar{Q} \phi$ processes by a simple replacement of the parton densities as well.

Acknowledgments The work was motivated by activities of the $L H C$ Higgs Cross Section Working Group. The author would like to thank Marco Bonvini, Stefan Liebler, and Frank Tackmann for constructive comments on the manuscript, and Marisa Sandhoff and Torsten Harenberg for administration of the DFG FUGG cluster at Bergische Universität Wuppertal, where most of the calculations for this paper were performed. Financial support by DFG, contract HA 2990/6-1 is greatly acknowledged.

Open Access This article is distributed under the terms of the Creative Commons Attribution 4.0 International License (http://creativecomm ons.org/licenses/by/4.0/), which permits unrestricted use, distribution, and reproduction in any medium, provided you give appropriate credit to the original author(s) and the source, provide a link to the Creative Commons license, and indicate if changes were made.

Funded by $\mathrm{SCOAP}^{3}$. 


\section{References}

1. J.F. Gunion, H.E. Haber, G.L. Kane, S. Dawson, The Higgs Hunter's guide. Front. Phys. 80, 1 (2000)

2. The LHC Higgs Cross Section Working Group Collaboration, Handbook of LHC Higgs Cross Sections: 1. Inclusive Observables. arXiv:1101.0593 [hep-ph]

3. The LHC Higgs Cross Section Working Group Collaboration, Handbook of LHC Higgs Cross Sections: 2. Differential Distributions. arXiv: 1201.3084

4. The LHC Higgs Cross Section Working Group Collaboration, Handbook of LHC Higgs Cross Sections: 3. Higgs Properties. arXiv: 1307.1347

5. C. Delaunay, T. Golling, G. Perez, Y. Soreq, Enhanced Higgs boson coupling to charm pairs. Phys. Rev. D 89, 033014 (2014). arXiv: 1310.7029

6. M.E. Gómez, S. Heinemeyer, M. Rehman, The Quark flavor violating Higgs decay $h \rightarrow \bar{b} s+b \bar{s}$ in the MSSM. arXiv:1511.04342

7. C. Buttar et al., Les Houches physics at TeV colliders 2005, standard model, $Q C D, E W$, and Higgs working group: Summary report. arXiv:hep-ph/0604120

8. R. Harlander, M. Krämer, M. Schumacher, Bottom-Quark Associated Higgs-Boson Production: Reconciling the Fourand Five-Flavour Scheme Approach. arXiv:1112.3478. https://twiki.cern.ch/twiki/pub/LHCPhysics/MSSMNeutral/ santandermatching-hks.pdf (LHC Higgs Cross Section Working Group Wiki Page)

9. S. Dittmaier, M. Krämer, M. Spira, Higgs radiation off bottom quarks at the Tevatron and the LHC. Phys. Rev. D 70, 074010 (2004). arXiv:hep-ph/0309204

10. S. Dawson, C.B. Jackson, L. Reina, D. Wackeroth, Exclusive Higgs boson production with bottom quarks at hadron colliders. Phys. Rev. D 69, 074027 (2004). arXiv:hep-ph/0311067

11. R.V. Harlander, W.B. Kilgore, Higgs boson production in bottom quark fusion at next-to-next-to-leading order. Phys. Rev. D 68, 013001 (2003). arXiv:hep-ph/0304035

12. F. Maltoni, G. Ridolfi, M. Ubiali, b-initiated processes at the LHC: a reappraisal. JHEP. 1207, 022 (2012). arXiv:1203.6393

13. S. Forte, D. Napoletano, M. Ubiali, Higgs production in bottomquark fusion in a matched scheme. Phys. Lett. B 751, 331 (2015). arXiv:1508.01529

14. M. Bonvini, A.S. Papanastasiou, F.J. Tackmann, Resummation and Matching of $b$-Quark Mass Effects in $b \bar{b} H$ Production. JHEP 1511, 196 (2015). arXiv:1508.03288

15. M. Wiesemann, R. Frederix, S. Frixione, V. Hirschi, F. Maltoni, P. Torrielli, Higgs production in association with bottom quarks. JHEP 1502, 132 (2015). arXiv: 1409.5301

16. R. Hamberg, W.L. van Neerven, T. Matsuura, A complete calculation of the order $\alpha_{s}^{2}$ correction to the Drell-Yan K factor. Nucl. Phys. B 359, 1343 (1991) (E) ibid.B 6442002403

17. R. Hamberg, W.L. van Neerven, T. Matsuura, A complete calculation of the order $\alpha_{s}^{2}$ correction to the Drell-Yan K factor. Nucl. Phys. B (E) 644, 403 (2002)

18. B.A. Kniehl, Associated production of Higgs and $Z$ bosons from gluon fusion in hadron collisions. Phys. Rev. D 42, 2253 (1990)

19. D.A. Dicus, C. Kao, Higgs Boson- $Z^{0}$ production from Gluon fusion. Phys. Rev. D 38, 1008 (1988)

20. D.A. Dicus, C. Kao, Higgs Boson- $Z^{0}$ production from Gluon fusion. Phys. Rev. D (E) 42, 412 (1990)
21. O. Brein, A. Djouadi, R. Harlander, NNLO CD corrections to the Higgs-Strahlung processes at hadron colliders. Phys. Lett. B 579, 149 (2004). arXiv:hep-ph/0307206

22. R.V. Harlander, S. Liebler, H. Mantler, SusHi: a program for the calculation of Higgs production in gluon fusion and bottom-quark annihilation in the Standard Model and the MSSM. Comput. Phys. Commun. 184, 1605 (2013). arXiv:1212.3249

23. E. Boos, T. Plehn, Higgs-boson production induced by bottom quarks. Phys. Rev. D 69, 094005 (2004). arXiv:hep-ph/0304034

24. F. Maltoni, Z. Sullivan, S. Willenbrock, Higgs-boson production via bottom-quark fusion. Phys. Rev. D 67, 093005 (2003). arXiv:hep-ph/0301033

25. D. Rainwater, M. Spira, D. Zeppenfeld, Higgs Boson Production at Hadron Colliders: Signal and Background Processes. arXiv:hep-ph/0203187

26. K.G. Chetyrkin, J.H. Kühn, M. Steinhauser, RunDec: a Mathematica package for running and decoupling of the strong coupling and quark masses. Comput. Phys. Commun. 133, 43 (2000). arXiv:hep-ph/0004189

27. A.D. Martin, W.J. Stirling, R.S. Thorne, G. Watt, Parton distributions for the LHC. Eur. Phys. J. C 63, 189 (2009). arXiv:0901.0002

28. A. Buckley, J. Ferrando, S. Lloyd, K. Nordström, B. Page, M. Rüfenacht, M. Schönherr, G. Watt, LHAPDF6: parton density access in the LHC precision era. Eur. Phys. J. C 75, 3 (2015). arXiv: 1412.7420

29. M.R. Whalley, D. Bourilkov, R.C. Group, The Les Houches Accord PDFs (LHAPDF) and LHAGLUE. arXiv:hep-ph/0508110. http:// projects.hepforge.org/lhapdf/

30. L.A. Harland-Lang, A.D. Martin, P. Motylinski, R.S. Thorne, Parton distributions in the LHC era: MMHT 2014 PDFs. Eur. Phys. J. C 75, 204 (2015). arXiv:1412.3989 [hep-ph]

31. S. Dulat et al., The CT14 Global Analysis of Quantum Chromodynamics. Phys. Rev. D 93(3), 033006 (2016). arXiv:1506.07443

32. R.D. Ball et al., [NNPDF Collaboration], Parton distributions for the LHC Run II. JHEP 1504, 040 (2015). arXiv: 1410.8849

33. E. Bagnaschi, R.V. Harlander, S. Liebler, H. Mantler, P. Slavich, A. Vicini, Towards precise predictions for Higgs-boson production in the MSSM. JHEP 1406, 167 (2014). arXiv:1404.0327

34. M. Krämer, Associated Higgs Production with Bottom Quarks at Hadron Colliders. arXiv:hep-ph/0407080

35. R. Harlander, Standard and SUSY Higgs production at the LHC. Pramana 67, 875 (2006). arXiv:hep-ph/0606095

36. T. Ahmed, N. Rana, V. Ravindran, Higgs boson production through $b \bar{b}$ annihilation at threshold in $\mathrm{N}^{3}$ LO QCD. JHEP 1410, 139 (2014). arXiv: 1408.0787

37. T. Ahmed, M.K. Mandal, N. Rana, V. Ravindran, Higgs rapidity distribution in $b \bar{b}$ annihilation at threshold in $\mathrm{N}^{3}$ LO QCD. JHEP 1502, 131 (2015). arXiv: 1411.5301

38. S. Buehler, F. Herzog, A. Lazopoulos, R. Mueller, The fully differential hadronic production of a Higgs boson via bottom quark fusion at NNLO. JHEP 1207, 115 (2012). arXiv:1204.4415

39. R.V. Harlander, A. Tripathi, M. Wiesemann, Higgs production in bottom quark annihilation: transverse momentum distribution at NNLO+NNLL. Phys. Rev. D 90, 015017 (2014). arXiv:1403.7196

40. R. Harlander, M. Wiesemann, Jet-veto in bottom-quark induced Higgs production at next-to-next-to-leading order. JHEP 1204, 066 (2012). arXiv:1111.2182

41. R.V. Harlander, K.J. Ozeren, M. Wiesemann, Higgs plus jet production in bottom quark annihilation at next-to-leading order. Phys. Lett. B 693, 269 (2010). arXiv:1007.5411 